\title{
REVIEW
}

\section{Boron neutron capture therapy assisted by boron-conjugated nanoparticles}

\author{
Shogo Sumitani ${ }^{1}$ and Yukio Nagasaki ${ }^{1,2,3}$
}

For high-performance boron neutron capture therapy (BNCT), core-shell-type biodegradable nanoparticles possessing boron clusters in their cores are designed to improve their accumulation tendency in the tumor region. The copolymerization of styrene carrying carborane with a poly(ethylene glycol)-block-poly(lactide) copolymer (PEG-b-PLA) that possesses an acetal group at its PEG end and a methacryloyl group at its PLA end (acetal-PEG- $b$-PLA-MA) was performed in an aqueous medium. As the acetal-PEG- $b$-PLA-MA forms a polymeric micelle in an aqueous medium, the carborane monomer in the hydrophobic core is solubilized, and the carborane in the core of the micelle is given covalent conjunction. Two carborane compounds, carborane with a mono-vinylbenzyl group [1-(4-vinylbenzyl)-closo-carborane (VB-carborane)] and carborane with di-vinylbenzyl groups [1,2-bis(4-vinylbenzyl)-closo-carborane ((VB) ${ }_{2}$-carborane)], were newly prepared and employed for the core polymerizations. The encapsulation efficiency of the VB-carborane in the micelles is much higher than that of the (VB) $2_{2}$ carborane. This improved efficiency is most likely caused by the interaction of the hydrogen bonding of the VB-carborane with the polymer micelle matrix, while no hydrogen bonding sites remain in the case of the (VB) $)_{2}$-carborane. The obtained corepolymerized and boron-conjugated micelles (PM, which were prepared from VB-carborane) showed extremely high stability under physiological conditions, which suppressed the leakage of boron compounds owing to the existence of the covalent bonds. The accumulation of the PM (7.2 ID\% per g) in tumors is much higher than that of the non-polymerized micelles (NPM) $(0.7 \mathrm{ID} \%$ per $\mathrm{g}$ ) under the same conditions (at $24 \mathrm{~h}$ after injection) because of the enhanced stability of the micelles under physiological conditions. In addition, thermal neutron irradiation experiments indicated significant suppression of the growth of tumor volume in tumor-bearing mice treated with the PM. It is important to note that the PM administered via intravenous injection were excreted almost completely from the major organs, except for the tumor, after a week. Therefore, these boronconjugated micelles represent a promising approach to the creation of a novel boron carrier for BNCT. Polymer Journal (2012) 44, 522-530; doi:10.1038/pj.2012.30; published online 4 April 2012

Keywords: biodistribution; boron neutron capture therapy; drug delivery systems; nanoparticle-assisted BNCT; polymeric micelles

\section{INTRODUCTION}

Boron neutron capture therapy (BNCT) has attracted significant attention as a selective and non-invasive type of cancer therapy. ${ }^{1,2}$ This therapy is based on the capture reaction of low-energy $(0.025 \mathrm{eV})$ thermal neutrons using nonradioactive boron-10 $\left({ }^{10} \mathrm{~B}\right)$, which produces $\alpha$ particles and ${ }^{7} \mathrm{Li}$ nuclei with high energy, as follows:

$$
\begin{gathered}
{ }^{10} \mathrm{~B}+\mathrm{n}_{\mathrm{th}}(0.025 \mathrm{eV}) \rightarrow\left[{ }^{11} \mathrm{~B}\right] \rightarrow{ }^{4} \mathrm{He}+{ }^{7} \mathrm{Li}+2.79 \mathrm{MeV}(6 \%) \\
\rightarrow{ }^{4} \mathrm{He}+{ }^{7} \mathrm{Li}+2.31 \mathrm{MeV}(94 \%) \\
\quad \downarrow \\
{ }^{7} \mathrm{Li}+\gamma+0.48 \mathrm{MeV}
\end{gathered}
$$

These high linear-energy-transfer particles dissipate their energy before traveling across the diameter of cells $(5-9 \mu \mathrm{m})$ within tissues, producing cytotoxic effects. The clinical potential of BNCT was proposed in the 1930s by Locher, ${ }^{3}$ and clinical trials were initiated in the 1950 s by Farr at the Brookhaven National Laboratory ${ }^{4,5}$ and by Sweet and Brownell in the 1970s at Massachusetts General Hospital using the Massachusetts Institute of Technology Reactor. ${ }^{6}$ However, the boron compounds had poor tumor retention, leading to low therapeutic efficacy. Among the hundreds of newly synthesized lowmolecular-weight boron-containing compounds, two appeared to be promising. One such compound, a polyhedral borane anion, was sodium borocaptate (BSH). ${ }^{7}$ The second was boronophenylalanine. ${ }^{8,9}$ These two compounds had low toxicity and attained tumor/blood boron ratios $(\mathrm{T} / \mathrm{B}$ ratios $)>1$, and they have been used clinically for BNCT of brain and other tissue tumors. Soloway et al. ${ }^{10}$ revealed that $\mathrm{BSH}$ has the potential to bind with brain tumors, and clinical studies using BSH for brain tumors were begun by Hatanaka. ${ }^{11,12}$ Meanwhile, Mishima et al. ${ }^{13}$ used boronophenylalanine to treat melanoma

${ }^{1}$ Department of Materials Science, Graduate School of Pure and Applied Sciences, University of Tsukuba, Ibaraki, Japan; ${ }^{2}$ Master's School of Medical Sciences, Graduate Schoo of Comprehensive Human Sciences, University of Tsukuba, Ibaraki, Japan and ${ }^{3}$ Satellite Laboratory, International Center for Materials Nanoarchitectonics (WPI-MANA), National Institute of Materials Science (NIMS), Ibaraki, Japan

Correspondence: Professor Y Nagasaki, Department of Materials Science, Graduate School of Pure and Applied Science, University of Tsukuba, Tennoudai 1-1-1, Tsukuba, Ibaraki 305-8573, Japan.

E-mail: yukio@nagalabo.jp

Received 18 January 2012; revised 9 February 2012; accepted 10 February 2012; published online 4 April 2012 
patients. However, because these compounds are rapidly cleared from the bloodstream (the half-life of blood-circulation time of BSH and boronophenylalanine is $\left.t_{1 / 2}<1 \mathrm{~h}\right),{ }^{14,15}$ a high dose of ${ }^{10} \mathrm{~B}$-compounds is generally required to allow a sufficient concentration of ${ }^{10} \mathrm{~B}$ atoms to accumulate in a tumor region. Therefore, the therapeutic value of cancer BNCT under in vivo conditions is largely dependent on the development of effective boron carrier systems that can achieve modulated disposition in the body through the intravenous route and facilitate accumulation in the tumor region. Recently, the development of boron delivery agents for BNCT has been studied to improve their accumulation tendency in the tumor region. The following requirements are important for successful boron delivery:

(i) normal tissue uptake with high tumor uptake and concomitantly high tumor/normal tissues and tumor/blood concentration ratio $(>3-4: 1)$

(ii) tumor concentrations of ca. $20 \mu \mathrm{g}{ }^{10} \mathrm{~B}$ per g;

(iii) rapid clearance from blood and normal tissues; and

(iv) no unexpected side effects without thermal neutron irradiation.

A promising strategy in this regard is the concept of drug delivery systems. ${ }^{16,17}$ Matsumura and Maeda et al. have reported that a drug delivery system based on nanomaterials, including proteins, drugconjugated polymers and nano-sized particles, enabled the accumulation of drugs in the tumor region because of the so-called 'enhanced permeability and retention' (EPR) effect. ${ }^{18,19}$ In this regard, various approaches have been employed for the targeting of ${ }^{10} \mathrm{~B}$ atoms into the tumor region based on the concepts that include the use of macromolecules such as boron-conjugated dendrimers and biological complexes, ${ }^{20,21}$ dextran conjugates, ${ }^{22}$ and hyaluronan conjugates. ${ }^{23}$ In particular, poly(ethylene glycol) (PEG)-modified liposomes encapsulating $\mathrm{BSH}$ in their interior have attracted attention as effective nano-sized carriers of ${ }^{10} \mathrm{~B}$ atoms because of their biocompatibility, long blood-circulation time and effective accumulation in tumors through EPR effects. ${ }^{24}$ Maruyama et al. prepared a transferrin-conjugated PEG-modified liposome encapsulating $\mathrm{BSH}$ to enhance the specificity of the liposome against tumors ${ }^{25-27}$ because it is well-known that the transferrin receptor concentration on tumor cells is much higher than that on normal cells. ${ }^{28}$ Although the PEGmodified liposomes encapsulating BSH showed higher accumulation in the tumor region than free BSH through the EPR effect, the therapeutic efficacy of the BSH-encapsulated liposomes remains controversial because of the leakage of the encapsulated BSH from the liposome into the blood stream caused by the high ion osmotic pressure of the BSH-loaded interior of the liposome. ${ }^{29}$ An alternative approach is represented by the ${ }^{10} \mathrm{~B}$-compound-conjugated liposomes fabricated by covalently linking a lipid (hydrophobic group) with a ${ }^{10} \mathrm{~B}$-compound (hydrophilic group) to suppress the leakage of the ${ }^{10} \mathrm{~B}$-compound into the bloodstream. ${ }^{30,31}$ The ${ }^{10} \mathrm{~B}$-compoundconjugated liposome achieved a sufficient high ${ }^{10} \mathrm{~B}$ concentration in the tumor, but the synthesis of the nido-carborane required complicated preparation steps. Additionally, serious acute toxicity was observed in vivo due to the cytotoxicity of nido-carborane, producing acute toxicity within one day at a dose of $6.0 \mathrm{mg}$ boron per kg. ${ }^{31}$ Nakamura et al..$^{32,33}$ developed ${ }^{10} \mathrm{~B}$-compound-conjugated liposomes using lipids that link with ${ }^{10} \mathrm{~B}$-enriched $\mathrm{BSH}$ by covalent bonds to reduce the cytotoxicity of the boron-conjugated liposomes. However, the ${ }^{10} \mathrm{~B}$-enriched $\mathrm{BSH}$-conjugated liposomes exhibited a short retention time in tumor tissues that was most likely caused by the insufficient stability of the liposomes. ${ }^{33}$ Thus, the development of delivery systems for ${ }^{10} \mathrm{~B}$-compounds that are safe and stable in the bloodstream without the leakage of ${ }^{10} \mathrm{~B}$-compounds is a promising approach to BNCT.
To improve the accumulation efficiency of nanoparticles in the tumor region by the EPR effect, the following goals should be met because the EPR effect works gradually up to several days:

(i) long circulation of nanoparticles in the bloodstream and

(ii) no leakage of the entrapped drug during the circulation.

Longer blood-circulation tendency generates higher accumulation of nanoparticles in the tumor region. Recently, a new class of the drug delivery systems has emerged that is based on nano-sized polymeric micelles with a core-shell structure formulated through the selfassembly of PEG-block-poly(D,L-lactide) (PLA) copolymer (PEG- $b$ PLA). ${ }^{34,35}$ These systems exhibit improved biocompatibility, nontoxicity, long blood-circulation time, ${ }^{36-38}$ and biodegradability. ${ }^{39,40}$ The hydrophobic PLA core is able to accommodate hydrophobic drugs, and the brush-like hydrophilic PEG shell prevents protein adsorption and subsequent non-specific uptake by the reticuloendothelial system after intravenous injection. However, one of the disadvantages of the use of polymeric micelles as a drug carrier is the leakage of the incorporated drug from the micelles during blood circulation. ${ }^{41-44}$ To solve this problem, many studies have focused on the stabilization of the micelles by introducing chemical linkages into either the shell or the core segment to avoid the loss of micelle integrity. ${ }^{45-48}$ We have also previously reported the preparation of core-polymerized PEG- $b$-PLA micelles that possess a methacryloyl group at the PLA chain end through radical polymerization. ${ }^{49,50}$ Eventually, the core-polymerized micelles exhibited high dispersibility (even after lyophilization), as well as high stability against dilution, temperature change and sodium dodecyl sulfate solution. Indeed, these above-mentioned approaches (polymerized and crosslinked micelles) are critical to the formulation of micelles that are resistant to the loss of micelle integrity. However, even with the use of crosslinked micelles, the leakage of drugs from the polymerized or crosslinked micelles is not completely suppressed because of the diffusion of the drug from the core to the bloodstream. ${ }^{47,51}$ To solve this problem, two approaches are suggested: the drugs can be conjugated with the micelles through chemical linkages, ${ }^{52-54}$ or functional groups compatible with the drugs can be introduced into the hydrophobic segments of the block copolymers to suppress the leakage of the incorporated drugs into the bloodstream. ${ }^{55-57}$ In general, micelles that are conjugated with anticancer drugs as cancer-specific carriers must release the drugs after accumulation in the tumor region, indicating that the structure of the linkage between the drugs and the micelles should be limited. In the case of BNCT, however, no release of the boron compounds from the micelles is required because the nucleic reaction of the boron compounds by thermal neutrons can take place even within the covalently conjugated micelle, and cellular damage can still be caused by the generated $\alpha$ particle and lithium. Thus, the covalent conjugation of the boron compound with the carriers is a suitable architecture for BNCT.

Recently, we designed and prepared boron-conjugated micelles by the free-radical copolymerization of a PEG- $b$-PLA block polymer bearing an acetal group at the PEG end and a methacryloyl group at the PLA end (acetal-PEG- $b$-PLA-MA) with polymerizable boron clusters (carborane). During the course of this polymerization study of polymer micelles, we found that the entrapping efficiency of the boron cluster in the core of the micelle is highly dependent on the structure of the boron cluster. The boron-conjugated micelles are expected to suppress the leakage of the boron compounds into the bloodstream because of the existence of covalent bonds between the boron compounds and the PLA core, leading to prolonged blood- 

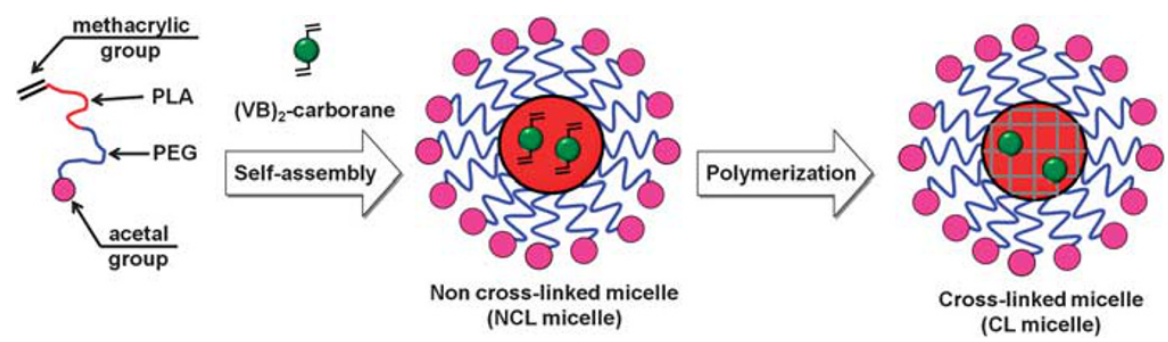

Figure 1 Schematic illustration of the preparation of non-crosslinked micelles (NCL) and crosslinked micelles (CL) micelles composed of acetalpoly(ethylene glycol)-block-poly(lactide) (PEG-b-PLA-MA and polymerizable carborane ((VB)2-carborane). This figure is reproduced from the study by Sumitani et al., ${ }^{59}$ by courtesy of the publishers, Elsevier, Amsterdam, The Netherlands.

circulation time and enhanced tumor accumulation. Additionally, the boron-conjugated micelles are expected to excrete easily from major organs via biodegradation of the PLA core. The boron-conjugated micelles are thus expected to exert significant therapeutic effects when used with thermal neutron irradiation in tumor-bearing mice because of the high concentration of boron atoms in the tumor region, which can be attributed to the high stability of the micelles in the bloodstream. This review introduces our work about the preparation and utilization of these boron-conjugated micelles for BNCT.

\section{CORE CROSSLINKED MICELLES}

We have designed and prepared a core crosslinked and boronconjugated micelle (CL micelle) by the radical copolymerization of acetal-PEG-b-PLA-MA with hydrophobic 1,2-bis(4-vinylbenzyl)-closocarborane $\left((\mathrm{VB})_{2}\right.$-carborane) as a crosslinker (Figure 1). The CL micelles enable suppression of the leakage of carborane and the loss of micelle integrity in the bloodstream, leading to a prolonged bloodcirculation time and an enhanced accumulation in the tumor region. The CL micelle is anticipated to gradually collapse through the biodegradation of the PLA segment after the therapy.

In accordance with the previous report, acetal-PEG- $b$-PLA-MA ${ }^{49}$ and $(\mathrm{VB})_{2}$-carborane were synthesized. ${ }^{58}$ To prepare the CL micelles, both $(\mathrm{VB})_{2}$-carborane and azobisisobutyronitrile are encapsulated into acetal-PEG- $b$-PLA-MA micelles, and the core of the micelles were thermally polymerized $\left(60^{\circ} \mathrm{C}\right.$ for $\left.24 \mathrm{~h}\right)$, as in our previous report. ${ }^{49}$ The average diameter and size distribution of the CL micelles $\left(85.3 \mathrm{~nm}, \quad \mu_{2} / \Gamma^{2}=0.171\right)$ were similar to those of the noncrosslinked micelles (NCL micelles) $\left(81.1 \mathrm{~nm}, \mu_{2} / \Gamma^{2}=0.158\right)$, suggesting that the core polymerization process does not influence the average diameter or size distribution of the micelles. The loading contents of the $(\mathrm{VB})_{2}$-carborane in the NCL and CL micelles were determined to be $1.0 \mathrm{wt} \%$ (loading efficiency: $3.2 \%$ ) and $0.9 \mathrm{wt} \%$ (loading efficiency: $3.1 \%$ ), respectively. The loading content of the carborane in the CL micelles is similar to that in NCL micelles, strongly indicating that the leakage of $(\mathrm{VB})_{2}$-carborane from the micelles does not occur at all during the polymerization process. However, the loading efficiency is not adequate in either case, which is most likely due to the low affinity of $(\mathrm{VB})_{2}$-carborane with the PLA forming the core of the micelle (see below).

The leakage of the encapsulated ${ }^{10} \mathrm{~B}$-compound from the nanosized carriers under physiological conditions in the presence of serum proteins is an important issue for in vivo applications. The stability of the incorporated drugs is simply determined by the dialysis method in vitro; viz., the amount of $(\mathrm{VB})_{2}$-carborane remaining in the micelle solution is determined based on the fluorescence intensity of the $(\mathrm{VB})_{2}$-carborane $\left(\lambda_{\mathrm{ex}}: 339 \mathrm{~nm}, \lambda_{\mathrm{em}}: 380 \mathrm{~nm}\right)$ after the micelles are mixed with phosphate-buffered saline $\left(0.2 \mathrm{mg} \mathrm{ml}^{-1}\right)$ in the presence

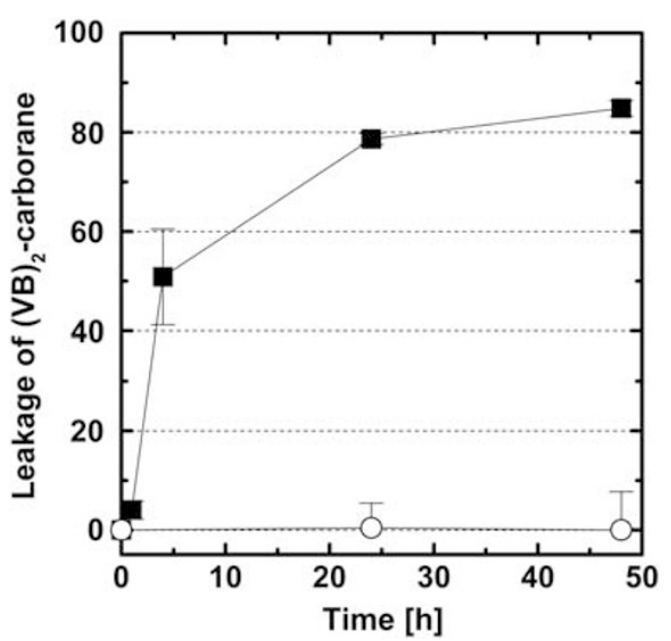

Figure 2 Time-dependent leakage of 1,2-bis(4-vinylbenzyl)-closo-carborane $(\mathrm{VB})_{2}$-carborane from non-crosslinked micelles (NCL) micelles (closed squares) and crosslinked micelles (CL) micelles (open circles) in the presence of $10 \%$ fetal bovine serum (FBS) at $37^{\circ} \mathrm{C}(n=3$, mean \pm s.d. $)$. This figure is reproduced from the study by Sumitani et al., ${ }^{59}$ by courtesy of the publishers, Elsevier, Amsterdam, The Netherlands.

of $10 \%$ fetal bovine serum (FBS). Figure 2 shows the time courses of the leakage of the $(\mathrm{VB})_{2}$-carborane from the $\mathrm{NCL}$ and the $\mathrm{CL}$ micelles. Significant amounts of $(\mathrm{VB})_{2}$-carborane were immediately leaked from the NCL micelles in the presence of FBS. There are several reports on the leakage of hydrophobic drugs from polymeric micelles in the presence of protein because of the loss of micelle integrity caused by the interaction between several blood components ( $\alpha$ - and $\beta$-globulin and/or BSA) and the micelles. ${ }^{42,44}$ However, the CL micelles showed no leakage of $(\mathrm{VB})_{2}$-carborane, even in the presence of FBS. The significantly reduced leakage of $(\mathrm{VB})_{2}$-carborane from the $\mathrm{CL}$ micelles occurred because of the existence of the crosslinking bonds between the $(\mathrm{VB})_{2}$-carborane and the PLA core.

The biodistributions of the boron species $\left((\mathrm{VB})_{2}\right.$-carborane) in the tumor-bearing mice using Inductively Coupled Plasma Mass Spectrometry (ICP-MS) at $24 \mathrm{~h}$ after injection of the CL or NCL micelles are shown in Figure 3. The boron concentrations in the blood and tumor after injection of the CL micelles (blood: $13.5 \%$ ID per g, tumor: $5.4 \%$ ID per g) were also significantly higher than the concentrations after the injection of the NCL micelles (blood: $1.8 \%$ ID per g, tumor: $1.4 \%$ ID per $\mathrm{g}$ ). These differences were most likely caused by the suppression of the leakage of the $(\mathrm{VB})_{2}$-carborane from the $\mathrm{CL}$ micelles in the bloodstream by the existence of the crosslinking bonds between the $(\mathrm{VB})_{2}$-carborane and the PLA core. Based on these 
results, our materials design of the covalent conjugation of the boron cluster to the micelle core is shown to represent a step forward for BNCT. ${ }^{59}$ However, the loading content of the carborane in the micelles is insufficient (ca. $1.0 \mathrm{wt} \%$ ), most likely due to the low compatibility of the carborane with the micelle core. From simple calculations using the pharmacokinetic data, a huge dose (2000-3000 mg kg-1) would be required to attain a therapeutically effective boron concentration in a tumor for BNCT (approximately 20 p.p.m. of ${ }^{10} \mathrm{~B}$ atoms per gram of tumor tissues) if the core
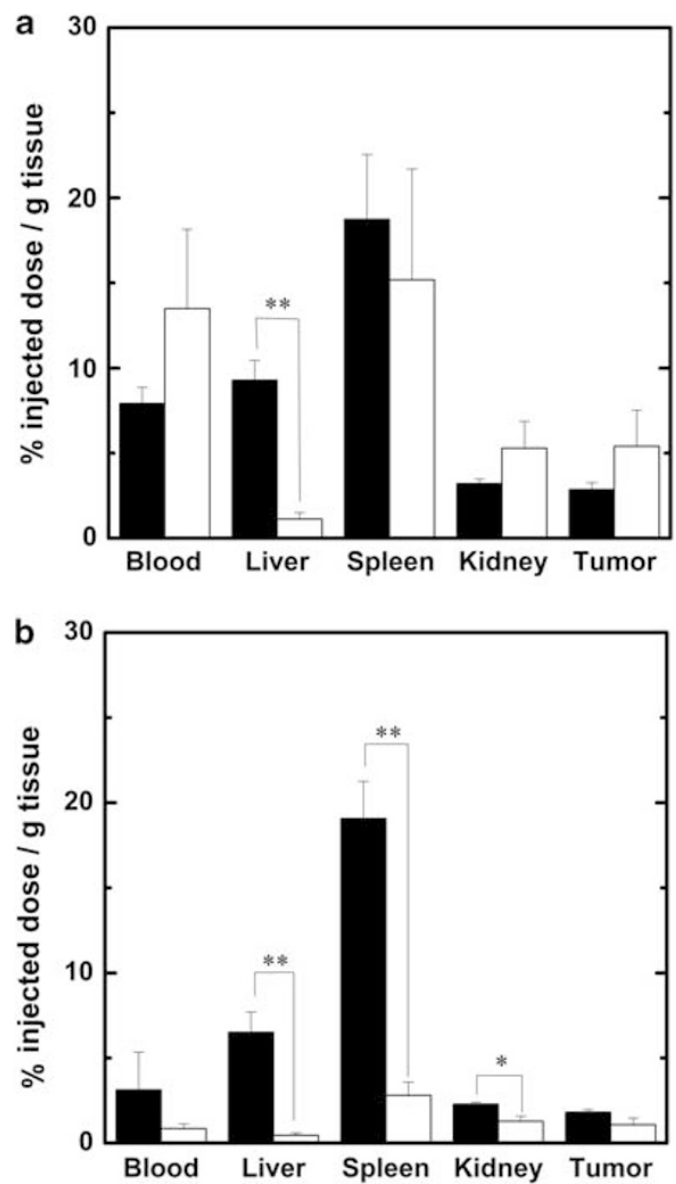

Figure 3 The comparison of the biodistribution between ${ }^{125}$-labeled micelles (black bars) and boron species (white bars) determined by radioactivity and ICP-MS, respectively, at $24 \mathrm{~h}$ after injection of CL micelles (a) and NCL micelles (b) into tumor-bearing mice $(n=4$, mean \pm s.d.). ${ }^{*} P<0.05,{ }^{* *} P<0.01$. This figure is reproduced from the study by Sumitani et al., 59 by courtesy of the publishers, Elsevier, Amsterdam, The Netherlands. crosslinked micelles were to be used. For this reason, the loading content of boron compounds in the micelles must be increased.

\section{CORE-POLYMERIZED AND BORON-CONJUGATED MICELLES}

As mono-vinylbenzyl-carborane (VB-carborane) retains one $\mathrm{CH}$ bond in its skeleton, some specific interactions with the micelle matrix are anticipated. Thus, VB-carborane was employed as a boron source instead of $(\mathrm{VB})_{2}$-carborane. The radical copolymerization of 1-(4-vinylbenzyl)-closo-carborane (VB-carborane) (Figure 4) in the core of the acetal-PEG- $b$-PLA-MA micelle produced core-polymerized micelles (PM) but not crosslinked micelles. Non-polymerized micelles (NPM ) were prepared by encapsulation of the VB-carborane into the acetal-PEG- $b$-PLA-MA micelles by means of the solvent evaporation method. ${ }^{59}$ The $\mathrm{PM}$ were prepared by the thermal polymerization $\left(60{ }^{\circ} \mathrm{C}\right.$ for $\left.24 \mathrm{~h}\right)$ after the encapsulation of both VB-carborane and azobisisobutyronitrile into the acetal-PEG- $b$-PLA-MA micelles by means of the solvent evaporation method. The average diameter $\left(67.3 \mathrm{~nm}, \mu_{2} / \Gamma^{2}=0.113\right)$ of the PM was similar to that of the NPM $\left(60.2 \mathrm{~nm}, \mu_{2} / \Gamma^{2}=0.119\right)$, suggesting that the core polymerization process does not influence the size distribution of the micelles. The loading contents of VB-carborane in the NPM and PM micelles were determined to be $8.5 \mathrm{wt} \%$ (loading efficiency: $23.5 \%$ ) and $7.7 \mathrm{wt} \%$ (loading efficiency: $21.5 \%$ ), respectively, as determined by Inductively Coupled Plasma Atomic Emission Spectroscopy (ICP-AES).

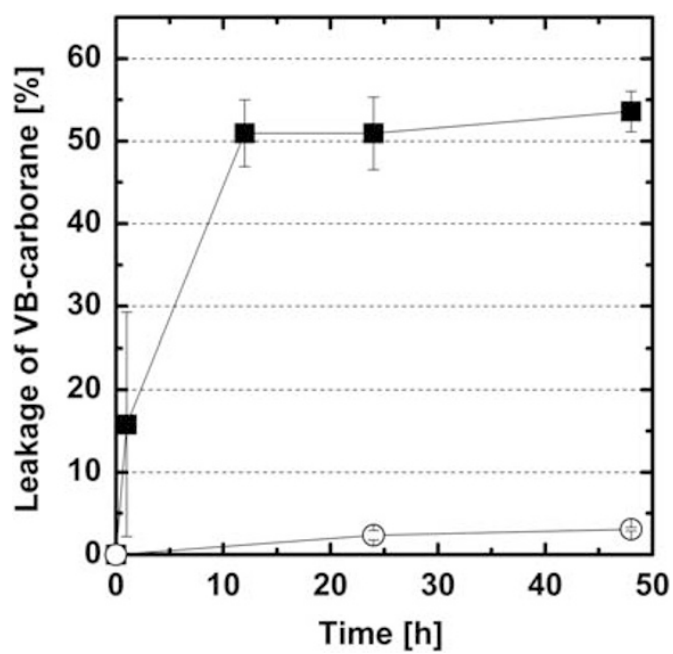

Figure 5 Time-dependent leakage of VB-carborane from non-polymerized micelles (NPM) (closed squares) and polymerized micelles (PM) (open circles) in the presence of $10 \%$ fetal bovine serum (FBS) at $37^{\circ} \mathrm{C}(n=3$, mean \pm s.d.). This figure is reproduced from the study by Sumitani et al.,, 1 by courtesy of the publishers, Elsevier, Amsterdam, The Netherlands.
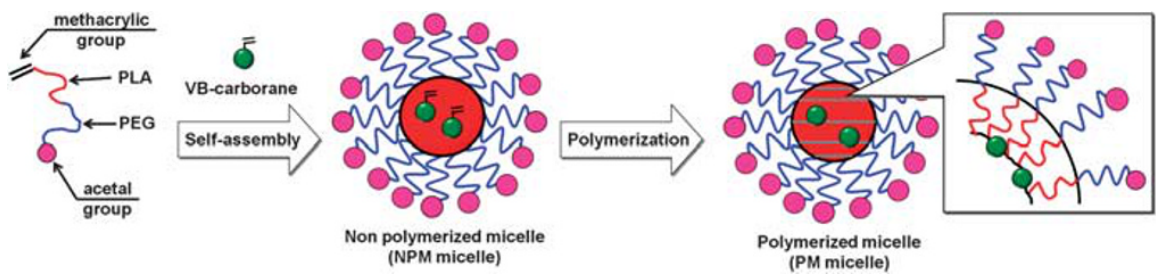

Figure 4 Schematic illustration of the preparation of non-polymerized micelles (NPM) and polymerized micelles (PM) composed of acetal-poly(ethylene glycol)-block-poly(lactide) (PEG-b-PLA-MA) and polymerizable (mono-functional) carborane (VB-carborane). This figure is reproduced from the study by Sumitani et al., ${ }^{61}$ by courtesy of the publishers, Elsevier, Amsterdam, The Netherlands. 

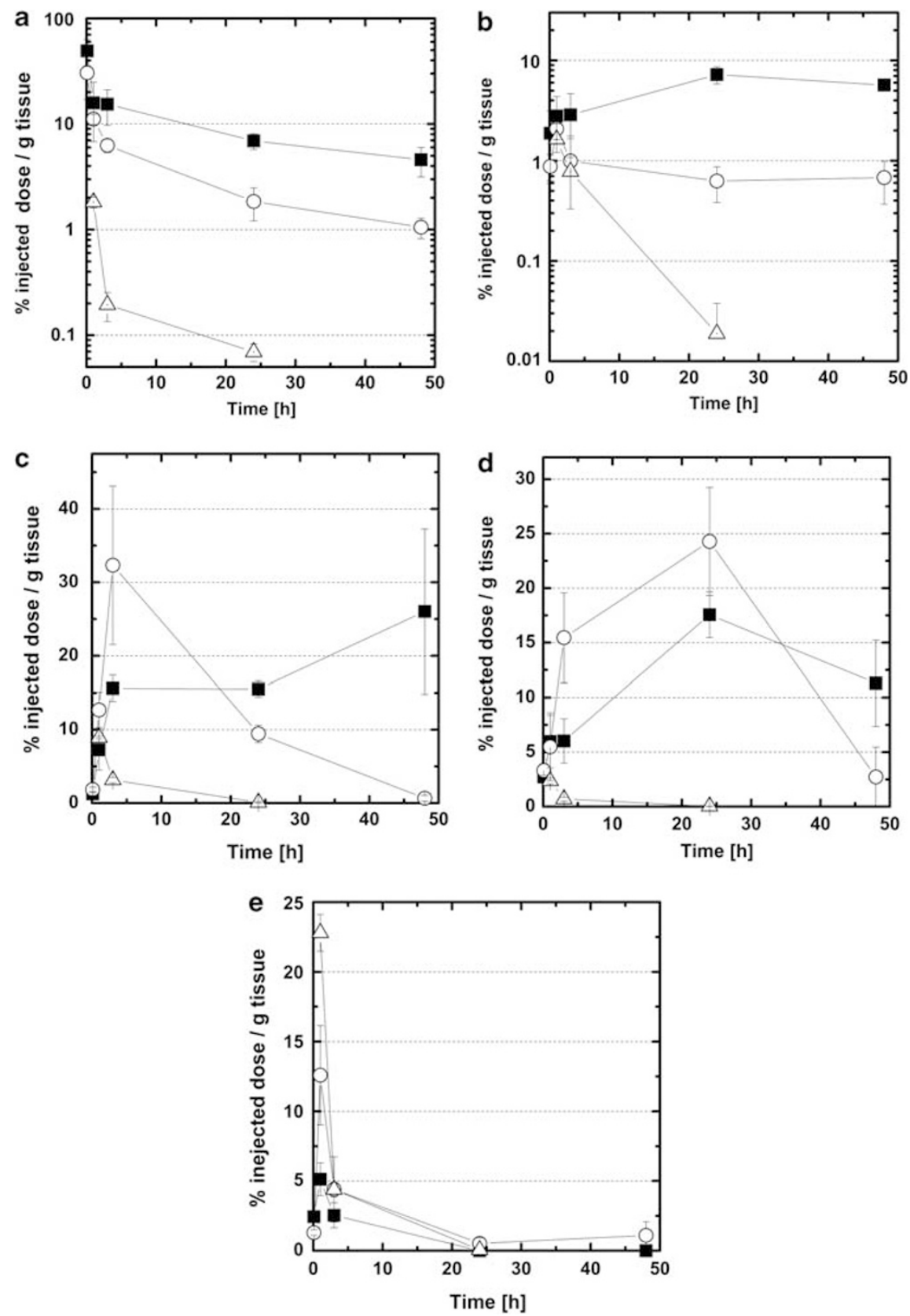

Figure 6 Tissue distribution profiles of boron species after injection of the polymerized micelles (PM) (closed squares), the non-polymerized micelles (NPM) (open circles), and free BSH (open triangles) in the blood (a), tumors (b), liver (c), spleen (d) and kidney (e) of the tumor-bearing mice, determined by ICPAES. The data are expressed as the mean \pm s.d. $(n=3)$. This figure is reproduced from the study by Sumitani et al., ${ }^{61}$ by courtesy of the publishers, Elsevier, Amsterdam, The Netherlands.

The loading contents of both micelles were comparable, indicating that the leakage of VB-carborane from the micelles does not occur during the polymerization process. Additionally, the loading content of VB-carborane in the acetal-PEG- $b$-PLA-MA micelles increased remarkably in comparison with that of the polymerizable carborane bearing two vinylbenzyl groups $\left((\mathrm{VB})_{2}\right.$-carborane), as described above. One of the likely reasons for this high loading efficiency is the hydrogen bond between the carborane and the PLA segments. The $\mathrm{CH}$ group of the carborane has been reported to form a $\mathrm{C}-\mathrm{H} \cdots \mathrm{O}=\mathrm{C}$ hydrogen bond with a carbonyl group $(\mathrm{C}=\mathrm{O}) ;{ }^{60}$ thus, the increased loading content of the VB-carborane in the PEG- $b$-PLA micelles is attributable to the hydrogen bonding of the VB-carborane with the PLA matrix in the micelle core. Indeed, the existence of the hydrogen bond between the $\mathrm{CH}$ group of the VB-carborane and the PLA segments in the micellar core was observed from the Fourier transform-infrared spectrum. ${ }^{61}$ The PM with high loading contents of boron compounds suppress the leakage of the boron compounds into the bloodstream because of the existence of the covalent bonds between the boron compounds and the PLA core, leading to prolonged blood-circulation time and enhanced tumor accumulation. Additionally, the PM are excreted easily from major organs via biodegradation of the PLA core. The PM exhibit significant 
therapeutic effects when used with thermal neutron irradiation in tumor-bearing mice because of the high concentration of boron atoms in the tumor, which can be attributed to the high stability of the micelles in the bloodstream. ${ }^{61}$

The leakage of VB-carborane from the PM and NPM under the physiological conditions is shown in Figure 5. This result shows that significant amounts (ca. 50\%) of VB-carborane leaked from the NPM after $12 \mathrm{~h}$ incubation. This leakage means that the stability of the VB-carborane physically entrapped in the acetal-PEG- $b$-PLA-MA micelles is insufficient under physiological conditions in the presence of serum proteins. In sharp contrast, the PM showed no leakage of the VB-carborane, even after $48 \mathrm{~h}$. This stability can most likely be attributed to the existence of covalent bonds between the VB-carborane and the PLA core. From these facts, we conclude that covalent conjugation, rather than physical entrapment, is required for in vivo stabilization in the case of $\mathrm{VB}$-carborane.

As described above, the distribution of boron species (carborane) in the CL and NCL micelles in tumor-bearing mice after intravenous injection differed, most likely because of the leakage of the carborane into the bloodstream. In this chapter, we describe the biodistribution of the boron species in the PM and NPM after intravenous injection into tumor-bearing mice. The distributions of boron species in the tissues of tumor-bearing mice injected with PM, NPM and free BSH evaluated using ICP-AES are shown in Figure 6. The concentrations of the boron atoms in each of the tissues and blood at specific times after intravenous injection are expressed as \%ID per g. The boron species of both NPM (1.1\% ID per g) and PM (4.6\% ID per g) remained in the bloodstream even $48 \mathrm{~h}$ after the injection, whereas over $99 \%$ of the injected dose of free BSH was immediately eliminated from the bloodstream by renal clearance because the amount of BSH that accumulated in the kidney $1 \mathrm{~h}$ after the injection (22.8\% ID per g) is apparently higher than that of both the NPM (12.6\% ID per g) and the PM (5.1\% ID per g) (Figures 6a and e). Additionally, these data indicate that the blood-circulation time of the boron species in the PM is prolonged compared with that of the NPM. The distribution of the boron species from the NPM in the kidney at $1 \mathrm{~h}$ after injection (12.6\% ID per g) was 2.5-fold higher than that of the PM (5.1\% ID per g) (Figure 6e). These data suggest that the VB-carborane leaks from the NPM easily, and it is eliminated from the bloodstream readily by renal clearance because of the lowmolecular weight of the VB-carborane, leading to the prolongation of the blood-circulation time of VB-carborane in the PM compared with its circulation time in the NPM. The accumulation level of boron species in the tumor tissues of the mice treated with the PM was $5.7 \% \mathrm{ID}$ per $\mathrm{g}$ at $48 \mathrm{~h}$ after the injection, whereas those of the $\mathrm{NPM}$ at $48 \mathrm{~h}$ and free BSH at $1 \mathrm{~h}$ after the injection were only $0.7 \% \mathrm{ID}$ per $g$ and $1.6 \%$ ID per $g$, respectively (Figure $6 \mathrm{~b}$ ). These results indicate that the accumulated amounts of boron species in tumor tissues are enhanced by the core-polymerization of the micelles because of the prolongation of the blood-circulation time of the VB-carborane. As the covalent conjugation of the core of the micelles via VB-carborane increases both the molecular weight of the matrix and the stability of the micelles, it is possible that the PM may remain in the body for a long time after administration. The long-term accumulation of certain types of nanoparticles in the body often causes unexpected toxicity. ${ }^{62}$ As shown in Figure 7, both boron species in the PM (a) and NPM (b) were almost completely eliminated from the major organs at 7 days after injection, as determined by ICP-AES measurements. Although the VB-carborane was conjugated covalently in the core of the PM, the near-complete excretion of the boron species was confirmed, which was most likely
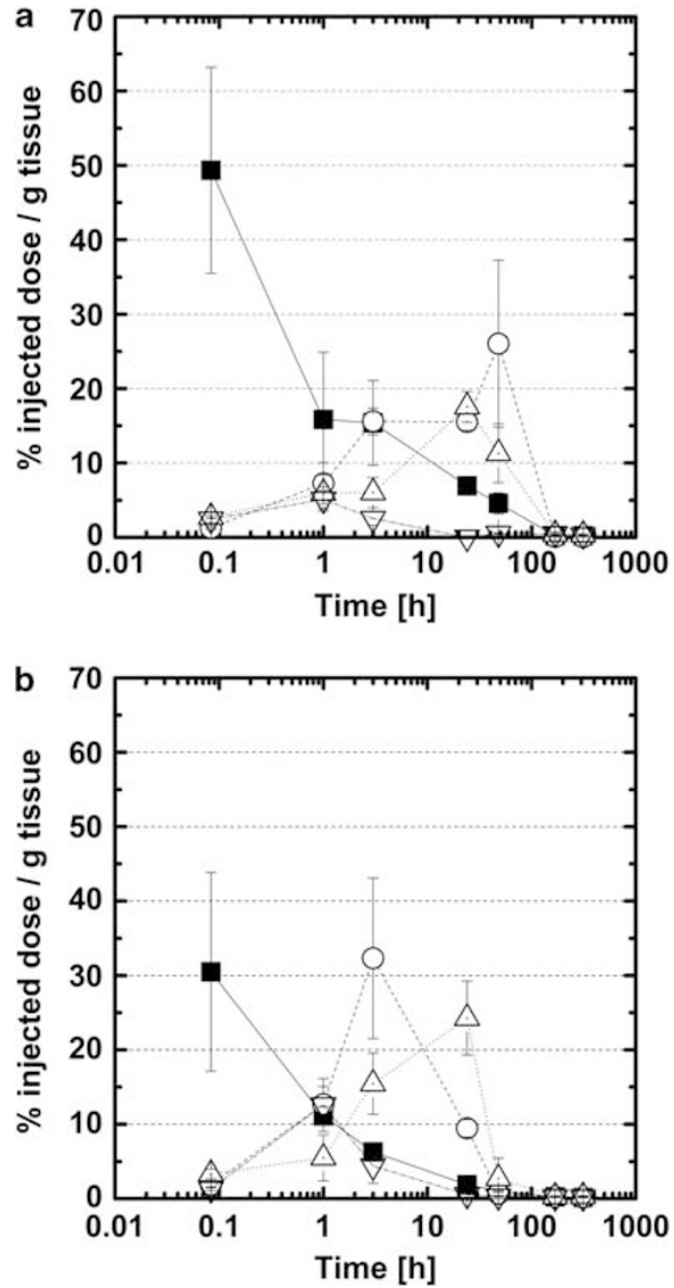

Figure 7 Long-term tissue distribution profile of boron species after injection of the polymerized micelles (PM) (a) and the non-polymerized micelles (NPM) (b) into the blood (closed squares), liver (open circles), spleen (open triangles) and kidney (open inverted triangles) of the normal mice, determined by ICP-AES. The data are expressed as the mean \pm s.d. $(n=4)$. This figure is reproduced from the study by Sumitani et al., ${ }^{61}$ by courtesy of the publishers, Elsevier, Amsterdam, The Netherlands.

caused by the hydrolysis of the ester linkage in the PLA segment. These results strongly suggest that the PM are useful not only for enhancing selective accumulation in tumor tissues but also for promoting the safety of the micelles. Thus, it can be concluded that the PM, which are constructed from acetal-PEG- $b$-PLA-MA and VB-carborane, seem to be suitable as an effective boron carrier for cancer BNCT.

Measurements of tumor growth after irradiation with thermal neutrons indicated the therapeutic effects of the ${ }^{10} \mathrm{~B}$-enriched $\mathrm{PM}$, NPM and BSH on BNCT. Figure $8 \mathrm{a}$ shows the changes in the tumor volume of mice bearing colon-26 tumors $(n=5)$ with thermal neutron irradiation for $37 \mathrm{~min}\left(1.6-1.8 \times 10^{12}\right.$ neutrons $\left.\mathrm{cm}^{-2}\right)$ after injection with ${ }^{10} \mathrm{~B}$-enriched $\mathrm{PM}$, NPM, free $\mathrm{BSH}$ or normal saline. The tumor growth in mice treated with the ${ }^{10} \mathrm{~B}$-enriched PM without irradiation was similar to that in the mice treated with normal saline with irradiation. This result indicates that the ${ }^{10} \mathrm{~B}$-enriched $\mathrm{PM}$ alone do not suppress the tumor growth. Additionally, no suppression of tumor growth was observed in the mice treated with both the ${ }^{10} \mathrm{~B}$-enriched NPM and BSH with irradiation by thermal neutrons 

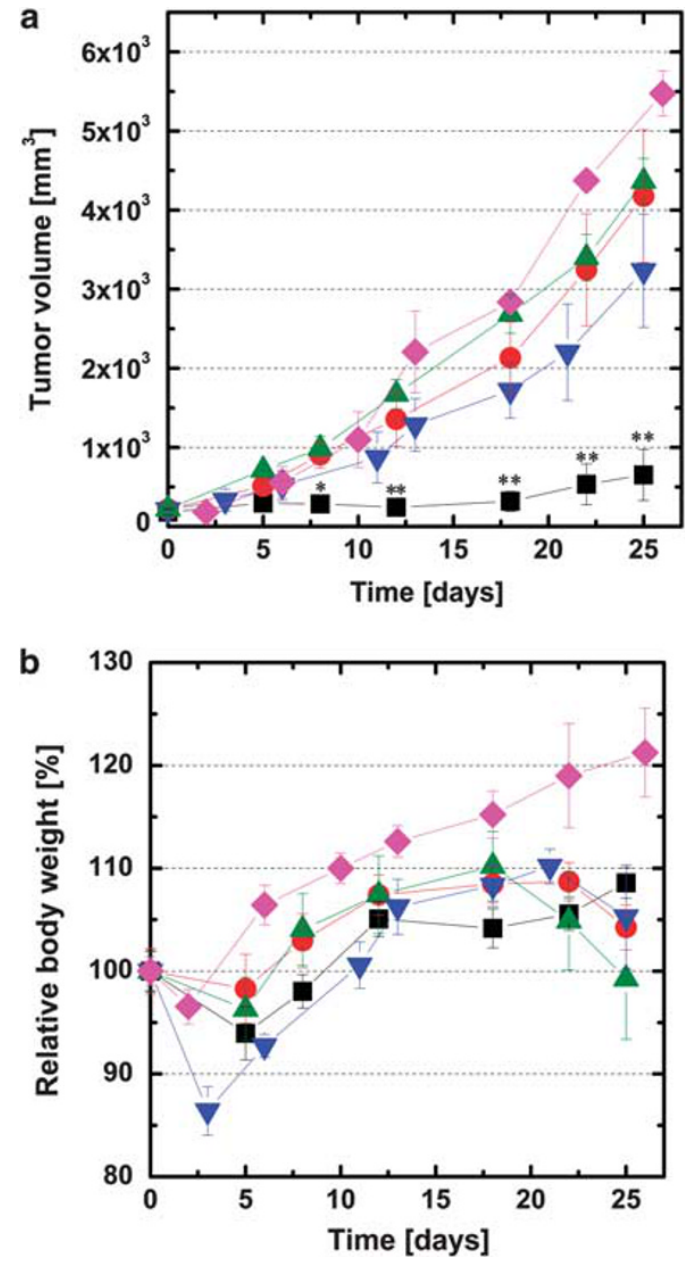

Figure 8 Tumor growth curve of tumor-bearing mice (a) or relative body weight change of tumor-bearing mice (b) after thermal neutron irradiation for $37 \mathrm{~min}\left(1.6-1.8 \times 10^{12}\right.$ neutrons $\left.\mathrm{cm}^{-2}\right)$ with injection of the ${ }^{10} \mathrm{~B}$-enriched PM (black squares), ${ }^{10} \mathrm{~B}$-enriched non-polymerized micelles (NPM) (red circles), or normal saline (green triangles) $24 \mathrm{~h}$ before irradiation, or sodium borocaptate $(\mathrm{BSH}) 1 \mathrm{~h}$ before irradiation (blue triangles). The ${ }^{10} \mathrm{~B}$-enriched PM were injected into the mice that were not irradiated (purple diamonds) as controls. The arrow indicates the day at which thermal neutron irradiation was performed. The data are expressed as the mean \pm s.e.m. $\left(n=5,{ }^{*} P<0.05,{ }^{*} P<0.01\right.$ against the control at the same time). This figure is reproduced from the study by Sumitani et al., ${ }^{61}$ by courtesy of the publishers, Elsevier, Amsterdam, The Netherlands.

due to the insufficient concentration of the ${ }^{10} \mathrm{~B}$ atoms in the tumor tissues. In sharp contrast, significant suppression of tumor growth was observed in the mice that were treated with the ${ }^{10} \mathrm{~B}$-enriched $\mathrm{PM}$ and received thermal neutron irradiation after 12 days $(P<0.01)$. Figure $8 \mathrm{~b}$ shows the time course of the body weight change in mice bearing colon-26 tumors that were subjected to thermal neutron irradiation for $37 \mathrm{~min}$ after treatment with ${ }^{10} \mathrm{~B}$-enriched $\mathrm{PM}$, ${ }^{10} \mathrm{~B}$-enriched $\mathrm{NPM}$, free $\mathrm{BSH}$ or normal saline. The mice treated with all micelle samples and normal saline showed no significant body weight loss compared with the starting weight. It should be noted that the ${ }^{10} \mathrm{~B}$-enriched PM and the ${ }^{10} \mathrm{~B}$-enriched NPM caused no acute toxicity, even at a dose of $15.6 \mathrm{mg} \mathrm{B}$ per $\mathrm{kg}$, although the previously reported liposomes composed of nido-carborane conjugated lipids showed acute toxicity within one day at a dose of $6.0 \mathrm{mg} \mathrm{B} \mathrm{per} \mathrm{kg.}{ }^{31}$ These facts strongly indicate that the ${ }^{10} \mathrm{~B}$-enriched PM surpass the liposomes composed of nido-carborane conjugated lipids as boron carriers for BNCT from the standpoint of toxicity. On the basis of these results, we conclude that the ${ }^{10} \mathrm{~B}$-enriched $\mathrm{PM}$ are suitable candidates for boron carriers in cancer BNCT, and that they exhibit beneficial therapeutic effects without any side effects.

\section{CONCLUSIONS}

We describe the preparation of CL micelles composed of acetal-PEG$b$-PLA-MA and $(\mathrm{VB})_{2}$-carborane. The leakage of $(\mathrm{VB})_{2}$-carborane from the micelles was suppressed, even in the presence of FBS, as a result of the introduction of crosslinking bonds between the $(\mathrm{VB})_{2-}$ carborane and the PLA core. Additionally, the CL micelles achieved a prolonged blood-circulation time and an enhanced accumulation in the tumor. In the next chapter, we describe the preparation of PM composed of acetal-PEG- $b$-PLA-MA with VB-carborane. The loading contents of the boron clusters (VB-carborane) in the micelles increased remarkably in comparison with those in the CL micelles. The leakage of the VB-carborane from the PM was completely suppressed, even in the presence of FBS, as a result of the introduction of covalent bonds between the carborane and the PLA core. Additionally, the PM exhibited a prolonged blood-circulation time and enhanced accumulation of boron species in the tumor. The ${ }^{10} \mathrm{~B}$-enriched PM showed remarkable therapeutic efficacy in BNCT; viz., selective and non-invasive BNCT was achieved. Therefore, these boron-conjugated micelles represent a promising approach to the creation of novel boron carriers for cancer BNCT.

\section{ACKNOWLEDGEMENTS}

This research was partially supported by a Grant-in-Aid for Scientific Research on Innovative Areas 'Molecular Soft-Interface Science' (no. 20106011), from the Ministry of Education, Culture, Sports, Science and Technology of Japan (MEXT). This work was performed using facilities of the Research Reactor Institute, Kyoto University and the Japan Atomic Energy Agency.

1 Barth, R. F., Soloway, A. H., Fairchild, R. G. \& Brugger, R. M. Boron neutron capture therapy of cancer: Realities and prospects. Cancer 70, 2995-3007 (1992).

2 Barth, R. F., Coderre, J. A., Vincente, G. H. \& Blue, T. E. Boron neutron capture therapy of cancer: Current status and future property. Cli. Cancer Res. 11, 3987-4002 (2005)

3 Locher, G. L. Biological effects and therapeutic possibilities of neutrons. Am. J. Roentgenol Radium Ther. 36, 1-13 (1936).

4 Farr, L. E., Sweet, W. H., Robertson, J. S., Foster, C. G., Locksley, H. B., Sutherland, D. L. Mendelsohn, M. L. \& Stickley, E. E. Neutron capture therapy with boron in the treatment of glioblastoma multiforme. Am. J. Roentgenol Radium Ther. Nucl. Med. 71, 279-291 (1954)

5 Godwin, J. T., Farr, L. E., Sweet, W. H. \& Robertson, J. S. Pathological study of eight patients with glioblastoma multiforme treated by neutroncapture therapy using boron10. Cancer 8, 601-615 (1955).

6 Asbury, A. K., Ojemann, Nielson, S. L. \& Sweet, W. H. Neuropathological study of fourteen cases of malignant brain tumor treated by boron-10 slow neutron capture therapy. J. Neuropathol Exp. Neurol. 31, 278-303 (1972).

7 Knoth, W. H., Sauer, J. C., England, D. C., Hertler, W. R. \& Murtterties, E. L. Chemistry of boranes. XIX. derivative chemistry of $\mathrm{B}_{10} \mathrm{H}_{10}{ }^{-2}$ and $\mathrm{B}_{12} \mathrm{H}_{12}{ }^{-2}$. J. Am. Chem. Soc. 86, 3973-3983 (1964).

8 Snyder, H. R., Reedy, A. J. \& Lennarz, W. Synthesis of aromatic boronic acids Aldehyde boronic acids and a boronic acid analog of tyrosine. J. Am. Chem. Soc. 80 835-838 (1958)

9 Roberts, D. C., Suda, K., Samanen, J. \& Kemp, D. S. Pluripotential amino acids. 1. (L)-p-dihydroxyborylphenylalanine (L-Bph) as a precursor of the L-Phe and L-Tyr containing peptides; specific tritiation of L-Phe containing peptides as a final step in synthesis. Tetrahedron Lett. 21, 3435-3438 (1989).

10 Soloway, A. H., Hatanaka, H. \& Davis, M. A. Penetration of brain and brain tumor. VII. Tumor-binding sulfhydryl boron compounds. J. Med. Chem. 10, 714-717 (1967).

11 Hatanaka, H. A revised boron-neutron capture therapy for malignant brain tumors: II. Interim clinical result with the patients excluding previous treatments. J. Neurol. 209, 81-94 (1975).

12 Hatanaka, H. \& Nakagawa, Y. Clinical results of long-surviving brain tumor patients who underwent boron neutron capture therapy. Int. J. Radiat. Oncol. Biol. Phys. 28, 1061-1066 (1994). 
13 Mishima, Y., Ichihashi, M., Hatta, S., Honda, C., Yamaura, K. \& Nakagawa, T. New thermal neutron capture therapy for malignant melanoma: melanogenesis-seeking ${ }^{10} \mathrm{~B}$ molecule-melanoma cell interaction from in vitro to first clinical trial. Pigment. Cell. Res. 2, 226-234 (1989).

14 Gibson, C. R., Staubus, A. E., Barth, R. F., Yang, W., Kleinholz, N. M., Jones, R. B., Church, K. B. G., Tjarks, W. \& Soloway, A. H. Electrospray ionization mass spectrometry coupled to reversed-phase ion-pair high-performance liquid chromatography for quantitation of sodium borocaptate and application to pharmacokinetic analysis. Anal. Chem. 74, 2394-2399 (2002).

15 Ichikawa, H., Taniguchi, E., Fujimoto, T. \& Fukumori, Y. Biodistribution of BPA and $\mathrm{BSH}$ after single, repeated and simultaneous administrations for neutron-capture therapy of cancer. Appl. Radiat. Isot. 67, 111-114 (2009).

16 Wu, G. 1., Barth, R. F., Yang, W., Lee, R., Tjarks, W., Backer, M. V. \& Backer, J. M. Boron containing macromolecules and nanovehicles as delivery agents for neutron capture therapy. Anticancer Agents Med. Chem. 6, 167-170 (2006).

17 Yanagie, H., Ogata, A., Sugiyama, H., Eriguchi, M., Takamoto, S. \& Takahashi, H. Application of drug delivery system to boron neutron capture therapy for cancer. Expert Opin. Drug. Deliv. 5, 427-443 (2008)

18 Matsumura, Y. \& Maeda, H. A new concept for macromolecular therapeutics in cancer chemotherapy: Mechanism of tumoritropic accumulation of proteins and the antitumor agent smancs. Cancer. Res. 46, 6387-6392 (1986).

19 Maeda, H., Sawa, T. \& Konno, T. Mechanism of tumor-targeted delivery of macromolecular drugs, including the EPR effect in solid tumor and clinical overview of the prototype polymeric drug SMANCS. J. Control Release. 74, 47-61 (2001).

20 Barth, R. F., Adams, D. M., Soloway, A. H., Alam, F. \& Darby, M. V. Boronated starburst dendrimer-monoclonal antibody immunoconjugates: Evaluation as a potential delivery system for neutron capture therapy. Bioconjug Chem. 5, 58-66 (1994).

21 Wu, G., Barth, R. F., Yang, W., Chatterjee, M., Tjarks, W., Ciesielski, M. J. \& Fenstermaker, R. A. Site-specific conjugation of boron-containing dendrimers to antiEGF receptor monoclonal antibody cetuximab (IMC-C225) and its evaluation as a potential delivery agent for neutron capture therapy. Bioconjug. Chem. 15, 185-194 (2004).

22 Gedda, L., Olsson, P., Ponten, J. \& Carlsson, J. Development and in vitro studies of epidermal growth factor-dextran conjugates for boron neutron capture therapy. Bioconjug. Chem. 7, 584-591 (1996).

23 Meo, C. D., Panza, L., Capitani, D., Mannia, L., Banzato, A., Rondina, M., Renier, D., Rosato, A. \& Crescenzi, V. Hyaluronan as Carrier of carboranes for tumor targeting in boron neutron capture therapy. Biomacromolecules 8, 552-559 (2007).

24 Mehta, S. C., Lai, J. C. K. \& Lu, D. R. Liposomal formulations containing sodium mercaptoundecahydrododecaborate (BSH) for boron neutron capture therapy J. Microencapsul. 13, 269-279 (1996).

25 Ishida, O., Maruyama, K., Tanahashi, H., Iwatsuru, M., Sasaki, K., Eriguchi, M. \& Yanagie, H. Liposomes bearing polyethyleneglycol-coupled transferrin with intracellular targeting property to the solid tumors in vivo. Pharm. Res. 18, 1042-1048 (2001).

26 Maruyama, K., Ishida, O., Kasaoka, S., Takizawa, T., Utoguchi, N., Shinohara, M., Chiba, M., Kobayashi, H., Eriguchi, M. \& Yanagie, H. Intracellular targeting of sodium mercaptoundecahydrododecaborate (BSH) to solid tumors by transferring-PEG liposomes, for boron neutron-capture therapy (BNCT). J. Control. Release. 98, 195-207 (2004).

27 Yanagie, H., Maruyama, K., Takizawa, T., Ishida, O., Ogura, K., Matsumoto, T., Sakurai, Y., Kobayashi, T., Shinohara, A., Rant, J., Skvarc, J., Ilic, R., Kuhne, G., Chiba, M., Furuya, Y., Sugiyama, H., Hisa, T., Ono, K., Kobayashi, H. \& Eriguchi, M. Application of boron-entrapped stealth liposomes to inhibition of growth of tumour cells in the in vivo boron neutron-capture therapy model. Biomed. Pharmacother. 60, 43-50 (2006).

28 Wagner, E., Curiel, D. \& Cotton, M. Delivery of drugs, proteins and genes into cells using transferring as a ligand for receptor-mediated endocytosis. Adv. Drug. Deliv. Rev. 14, 113-135 (1994).

29 Feakes, D. A., Shelly, K. \& Hawthorne, M. F. Selective boron delivery to murine tumors by lipophilic species incorporated in the membranes of unilamellar liposomes. Proc. Natl. Acad. Sci. USA 92, 1367-1370 (1995).

30 Miyajima, Y., Nakamura, H., Kuwata, Y., Lee, J. D., Masunaga, S., Ono, K. \& Maruyama, K. Transferrin-loaded nido-carborane liposomes: tumor-targeting boron delivery system for neutron capture therapy. Bioconjug. Chem. 17, 1314-1320 (2006).

$31 \mathrm{Li}, \mathrm{T}$., Hamdi, J. \& Hawthorne, M. F. Unilamellar liposomes with enhanced boron content. Bioconjug. Chem. 17, 15-20 (2006).

32 Lee, J. D., Ueno, M., Miyajima, Y. \& Nakamura, H. Synthesis of boron cluster lipids: closo-dodecaborate as an alternative hydrophilic function of boronated liposomes for neutron capture therapy. Org. Lett. 9, 323-326 (2007).

33 Ueno, M., Ban, H. S., Nakai, K., Inomata, R., Kaneda, Y., Matsumura, A. \& Nakamura, $\mathrm{H}$. Dodecaborate lipid liposomes as new vehicles for boron delivery system of neutron capture therapy. Bioorg. Med. Chem. 18, 3059-3065 (2010).

34 Kataoka, K., Harada, A. \& Nagasaki, Y. Block copolymer micelles for drug delivery: design characterization and biological significance. Adv. Drug. Dliv. Rev. 47, 113-131 (2001).

35 Gaucher, G., Dufresne, M. H., Sant, V. P., Kang, N., Maysinger, D. \& Leroux, J. C. Block copolymer micelles: preparation, characterization and application in drug delivery. J. Control Release 109, 169-199 (2005).

36 Verrechia, T., Spenlehauer, G., Bazile, D. V., Murry-Brelier, A., Archimbaud, Y. \& Veillard, M. Non-stealth (poly(lactic acid/albumin)) and stealth (poly(lactic acid-polyethylene glycol)) nanoparticles as injectable drug carriers. J. Control. Release 36, 49-61 (1995).

37 Hagan, S. A., Coombes, A. G. A., Garnett, M. C., Dunn, S. E., Davies, M. C., Illum, L., Davis, S. S., Harding, S. E., Purkiss, S. \& Gellert, P. R. Polylactide-poly(ethylene glycol) copolymers as drug delivery systems. 1. characterization of water dispersible micelle-forming systems. Langmuir. 12, 2153-2161 (1996).

38 Yamamoto, Y., Nagasaki, Y., Kato, Y., Sugiyama, Y. \& Kataoka, K. Long-circulating poly(ethylene glycol)-poly(D,L-lactide) block copolymer micelles with modulated surface charge. J. Control. Release. 77, 27-38 (2001)

39 Stefani, M., Coudane, J. \& Vert, M. In vitro ageing and degradation of PEG-PLA diblock copolymer-based nanoparticles. Polym. Degrad. Stab. 91, 2554-2559 (2006).

40 Yang, L., Ghzaoui, A. E. \& Li, S. In vitro degradation behavior of Poly(lactide)Poly(ethylene glycol) block copolymer micelles in aqueous solution. Int. J. Pharm. 400, 96-103 (2010).

41 Kim, S., Shi, Y., Kim, J. Y., Park, K. \& Cheng, J. X. Overcoming the barriers in micellar drug delivery: loading efficiency, in vivo stability, and micelle-cell interaction. Expert Opin. Drug. Deliv. 7, 49-62 (2010).

42 Chen, H., Kim, S., He, W., Wang, H., Low, P. S., Park, K. \& Cheng, J. X. Fast release of lipophilic agents from circulation PEG-PDLLA micelles revealed by in vivo förster resonance energy transfer imaging. Langmuir. 24, 5213-5217 (2008).

43 Burt, H. M., Zhang, X., Toleikis, P., Embree, L. \& Hunter, W. L. Development of copolymers of poly(D,L-lactide) and methoxypolyethylene glycol as micellar carriers of paclitaxel. Colloids Surf. B. Biointerfaces 16, 161-171 (1999).

44 Diezi, T. A., Bae, Y. \& Kwon, G. S. Enhanced Stability of PEG-block-poly(N-hexyl stearate L-aspartamide) micelles in the presence of serum proteins. Mol. Pharm. 7, 1355-1360 (2010)

45 Rösler, A., Vandermeulen, G. W. \& Klok, H. A. Advanced drug delivery devices via selfassembly of amphiphilic block copolymers. Adv. Drug. Deliv. Rev. 53, 95-108 (2001).

46 O'Reilly, R. K., Hawker, C. J. \& Wooley, K. L. Cross-linked block copolymer micelles: functional nanostructures of great potential and versatility. Chem. Soc. Rev. 35, 10681083 (2006).

47 Shuai, X., Merdan, T., Schaper, A. K., Xi, F. \& Kissel, T. Core-cross-linked polymeric micelles as paclitaxel carriers. Bioconjug. Chem. 15, 441-448 (2004).

48 Rijcken, C. J., Snel, C. J., Schiffelers, R. M., Nostrum, C. F. \& Hennink, W. E. Hydrolysable core-crosslinked thermosensitive polymeric micelles: synthesis, characterization and in vivo studies. Biomaterials 28, 5581-5593 (2007).

49 lijima, M., Nagasaki, Y., Okada, T., Kato, M. \& Kataoka, K. Core-polymerized reactive micelles from heterotelechelic amphiphilic block copolymers. Macromolecules 32, 1140-1146 (1999).

50 Kim, J. H., Emoto, K., Iijima, M., Nagasaki, Y., Aoyagi, T., Okano, T., Sakurai, Y. \& Kataoka, K. Core-stabilized polymeric micelle as potential drug carrier: increased solubilization of taxol. Polym. Adv. Technol. 10, 647-654 (1999).

$51 \mathrm{Du}$, W., Nyström, A. M., Zhang, K., Leonard, J. R. \& Wooley, K. L. ${ }^{19} \mathrm{~F}-$ and fluorescently labeled micelles as nanoscopic assemblies for chemotherapeutic delivery. Bioconjug. Chem. 19, 2492-2498 (2008)

52 Yokoyama, M., Okano, T., Sakurai, Y., Ekimoto, H., Shibazaki, C. \& Kataoka, K. Toxicity and antitumor activity against solid tumors of micelle-forming polymeric anticancer drug and its extremely long circulation in blood. Cancer Res. 51, 3229-3236 (1991).

53 Yoo, H. S., Lee, E. A. \& Park, T. G. Doxorubicin-conjugated biodegradable polymeric micelles having acid-cleavable linkage. J. Control Release 82, 17-27 (2002).

54 Bae, Y., Fukushima, S., Harada, A. \& Kataoka, K. Design of environment-sensitive supramolecular assemblies for intercellular drug delivery: polymeric micelles that are responsive to intracellular pH change. Angew. Chem. Int. Ed. Engl. 42, 4640-4643 (2003).

55 Kataoka, K., Matsumoto, T., Yokoyama, M., Okano, T., Sakurai, Y., Fukushima, S., Okamoto, K. \& Kwon, G. S. Doxorubicin-loaded poly(ethylene glycol)-poly( $\beta$-benzyl-Laspartate) copolymer micelles: Their pharmaceutical characteristics and biological significance. J. Control Release 64, 143-153 (2000).

56 Lee, J., Cho, E. C. \& Cho, K. Incorporation and release behavior of hydrophobic drug in functionalized poly(D,L-lactide)-block-poly(ethylene oxide) micelles. J. Control Release 94, 323-335 (2004)

57 Danquah, M., Fujiwara, T. \& Mahato, R. I. Self-assembling Methoxypoly(ethylene glycol)-b-poly(carbonate-co-L-lactide) block copolymer for drug delivery. Biomaterials 31, 2358-2370 (2010).

58 Benhabbour, S. R., Parott, M. C., Gratton, E. A. \& Adronov, A. Synthesis and properties of carborane-containing dendronized polymers. Macromolecules 40, 5678-5688 (2007).

59 Sumitani, S., Oishi, M. \& Nagasaki, Y. Carborane confined nanoparticles for boron neutron capture therapy: improved stability, blood circulation time and tumor accumulation. React Funct. Polym. 71, 684-693 (2011).

60 Davidson, M. G., Hibbert, T. G., Howard, J. A. K., Mackinnon, A. \& Wade, K. Definitive crystal structures of ortho-, meta- and para-carboranes: supramolecular structures directed solely by $\mathrm{C}-\mathrm{H} \cdots \mathrm{O}$ hydrogen bonding to hmpa (hmpa= hexamethylphosphoramide). Chem. Commun. 19, 2285-2286 (1996).

61 Sumitani, S., Oishi, M., Yaguchi, T., Murotani, H., Horiguchi, Y., Suzuki, M., Ono, K., Yanagie, H. \& Nagasaki, Y. Pharmacokinetics of core-polymerized, boron-conjugated micelles designed for boron neutron capture therapy for cancer. Biomaterials 33, 3568-3577 (2012).

62 Lewinski, N., Colvin, V. \& Drezek, R. Cytotoxicity of nanoparticles. Small 4, 26-49 (2008). 

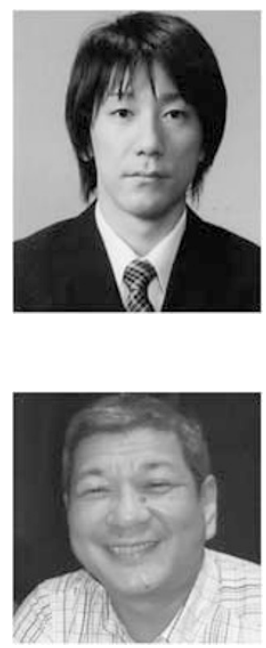

Shogo Sumitani was born in 1984. He received a BS degree in Engineering from the University of Tsukuba in 2007, and then studied under the direction of Professor Yukio Nagasaki at the University of Tsukuba, obtaining a MS degree in Engineering in 2009. He is currently pursuing his PhD studies under a supervision of Prof. Yukio Nagasaki from 2009, and has been a research fellow of the Japan Society for the Promotion of Science (JSPS) since 2010. During his doctoral studies, he studied at Université de Montréal as a visiting scientist. His research interests concern polymer chemistry, biomaterials, and drug delivery system.

Yukio Nagasaki was born in 1959. He received BS and $\mathrm{PhD}$ degrees from the Engineering School of Science, University of Tokyo, in 1982 and 1987. Since 1987, he was working at the Science University of Tokyo as Research Associate, Assistant Professor, Associate Professor and Professor. In 2004, he moved to the Graduate School of Pure and Applied Sciences, University of Tsukuba. He holds concurrent posts of Adjunct Professor, Master's School of Medical Sciences, Graduate School of Comprehensive Human Sciences, University of Tsukuba, Principal Investigator, International Center for Materials Nanoarchitectonics Satellite (WPI-MANA), National Institute for Materials Science (NIMS) and Principal Investigator, Strategic Initiatives (Project type), University of Tsukuba. During the last 30 years, he was engaged in materials science especially in the field of biology, pharmaceutics and medical science. He especially focused on biointerface and bionanoparticles. He published more than 250 scientific papers. He received the excellent $\mathrm{PhD}$ thesis award from Inoue Foundation of Science in 1989, Young Researcher Award from Polymer Society, Japan in 1993 and SPSJ Mitsubishi Chemical Award from Polymer Society, Japan in 2010. 